\title{
Superior oblique tendon (Brown's) syndrome as the presenting finding in childhood onset HLA-B27-related enthesitis and juvenile idiopathic oligoarticular arthritis
}

\author{
C. Pham ${ }^{1}$, V. Utz ${ }^{2}$, A. Marcotty², A. Zeft ${ }^{3}$, P. Rychwalski² \\ ${ }^{1}$ Case Western Reserve University; \\ Cole Eye Institute, Cleveland Clinic; \\ ${ }^{3}$ Division of Rheumatology, Pediatric Institute, Cleveland Clinic, Cleveland, OH, USA
}

\begin{abstract}
We report two patients who presented with Brown's syndrome. The first is a 7-year-old boy who at the time of his diagnosis was also found to have enthesitis and HLA-B27 positivity. The second patient was diagnosed with bilateral Brown's syndrome at 13 months of age. At age 7 she developed a persistent oligoarticular arthritis and unilateral anterior iritis consistent with the oligoarticular Juvenile Idiopatic Arthritis (JIA) phenotype. These cases highlight ophthalmologic findings and diagnostic considerations with respect to Brown's syndrome and associated childhood onset rheumatologic disease.
\end{abstract}

Key words: Brown's syndrome, Spondyloarthropathy, HLA-B27, Enthesitis, Juvenile arthritis.

Reumatismo, 2014; 66 (3): 240-244

\section{INTRODUCTION}

rown's syndrome is an ocular motility

disorder characterized by the inability to elevate the affected eye in adduction (1). It may have a congenital or acquired etiology. The spondyloarthropathies are a group of disorders that include ankylosing spondylitis, reactive arthritis, psoriatic arthritis, spondyloarthropathy associated with inflammatory bowel disease, and undifferentiated spondyloarthropathy (2). These disorders are further characterized by a negative rheumatoid factor, HLA-B27 positivity, and enthesitis. The ocular manifestation most commonly associated with these disorders is acute anterior uveitis. Juvenile idiopathic arthritis (JIA) is a heterogeneous group of diseases characterized by synovial inflammation and is the most common chronic rheumatic disease in children (3). Children with the oligoarticular subtype of JIA may develop an indolent anterior iritis, diagnosed with the aid of a screening ophthalmologic slit lamp examination.
We report two patients who presented with Brown's syndrome. The first is a 7-yearold boy who at the time of his diagnosis was also found to have enthesitis and HLAB27 positivity. The second patient was diagnosed with bilateral Brown's syndrome at 13 months of age. At age 7 she developed a persistent oligoarticular arthritis and unilateral anterior iritis consistent with the oligoarticular JIA phenotype. These cases highlight ophthalmologic findings and diagnostic considerations with respect to Brown's syndrome and associated childhood onset rheumatologic disease.

\section{CASE REPORTS}

\section{Case 1}

A 7-year-old boy with history of accommodative esotropia, amblyopia of the right eye, and anisometropia was referred to our institution for an ocular motility evaluation. His primary ophthalmologist noted an interval change in ocular motility, notably a 
small left hypertropia in primary and an inability to elevate the right eye in adduction. Previously this eye movement disorder had not been present, which was consistent with an acquired Brown syndrome. The patient also had a 3-month history of recurrent fever with headache, mild lymphadenopathy, and heel pain. Laboratory testing revealed an elevated erythrocyte sedimentation rate (ESR) of $91 \mathrm{~mm} / \mathrm{h}$, while his C-reactive protein, complete blood count, ferritin, anti-streptolysin $\mathrm{O}$ titer, and rheumatoid factor were within normal limits. Magnetic resonance imaging (MRI) of the orbits ordered by the referring physician was equivocal for trochlear inflammation. He was referred to our institution for both pediatric ophthalmologic and rheumatologic evaluations.

On presentation, the patient did not complain of diplopia, tenderness to palpation of the medial preorbital area, or pain with extraocular movements. His best-corrected visual acuity was 20/30 oculus dexter (OD) and 20/20 oculus sinister (OS). Motor examination with correction demonstrated excellent control of accommodative esotropia at distance and near fixation. A small left hypotropia was noted in primary, which increased to 8 prism diopter in left gaze with orthophoria in right gaze. Motility examination revealed limitation in elevation in adduction OD, with downshoot and normal motility in abduction OD (Fig. 1). No com- pensatory head tilt was noted. Slit lamp and posterior segment examinations were unremarkable. Cycloplegic refraction was $+8.75+0.75 \times 005$ OD and +5.75+1.00x 170 OS. We elected not to repeat dedicated orbital imaging given the constellation of clinical and laboratory findings supporting the diagnosis of Brown's syndrome.

Rheumatologic evaluation revealed swelling at the right Achilles tendon calcaneal insertion, consistent with enthesitis. There was no objective evidence of arthritis on physical exam. Further laboratory studies revealed a positive HLA-B27, negative antinuclear antibodies, and normal urine analysis. His repeat ESR was again high (92 $\mathrm{mm} / \mathrm{h}$ ), confirming the prior result. The patient was placed on an 8-week prednisone taper and weekly oral methotrexate $(0.5 \mathrm{mg} / \mathrm{kg} / \mathrm{dose})$ for the treatment of HLA-B27 related enthesitis. Four months later at a combined clinic follow-up, the patient's calcaneal enthesitis had resolved, he was afebrile, and his ESR was virtually normal ( 11 , normal $<10 \mathrm{~mm} / \mathrm{h}$ ), however, he remained with hypotropia.

\section{Case 2}

The second case is a 13 month old girl who presented to the pediatric ophthalmologist with a reported history of strabismus and lazy eye. On examination she had a chin up head posture and a left gaze preference with findings of bilateral under action of

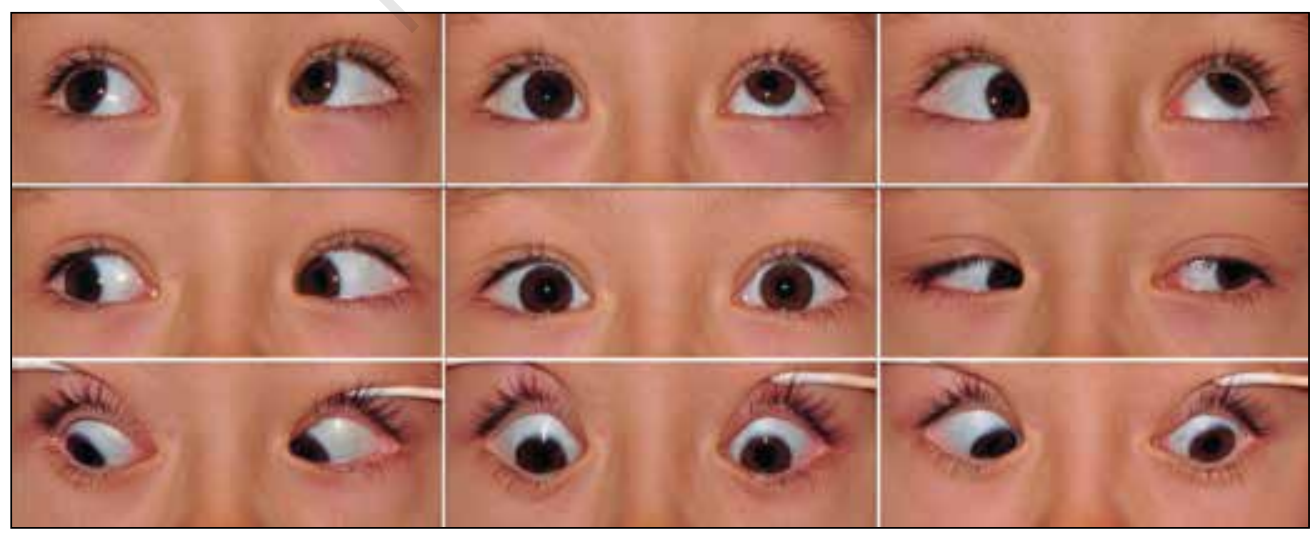

Figure 1 - Brown syndrome. Selected gaze positions of a 7-year-old with HLA-B27 positive enthesitis. The patient's eyes were straight in primary position. The motility evaluation revealed a right hypotropia in upgaze, which is more pronounced in adduction of the right eye in left upgaze. 
both inferior oblique muscles consistent with bilateral Brown syndrome. Her chin up head preference continued, but her examination at 28 months of age revealed that her Brown syndrome was now unilateral. Her eye movements remained limited in the direction of the left inferior oblique with improved ocular rotation of the right inferior oblique. At 7 years she developed oligoarticular arthritis. Her knee was treated with an intraarticular corticosteroid injection, which then required a second injection 4 years later to treat an arthritis flare. At the time of her arthritis flare, intraocular inflammation within her left anterior chamber was identified by screening ophthalmologic slit lamp examination. Her iritis resolved with a graduated course of topical prednisolone acetate treatment and to date has not recurred.

\section{DISCUSSION}

Brown's syndrome is a congenital or an acquired disorder of ocular motility characterized by the inability to elevate the affected eye in adduction $(4,5)$. Brown's original study reported 23 of 126 cases of simulated superior oblique tendon sheath syndrome, termed by Brown because the simulated fibrous sheath could not elongate. Five of the 23 he considered acquired cases, not congenital in origin, and 9 of the 23 he labeled intermittent. In his paper he speculated that the etiology of both intermittent and acquired cases had an underlying inflammatory etiology (4). A later retrospective case series performed by Wright et al., examined 85 Brown's syndrome patients. Forty seven of 85 cases $(55 \%)$ were labeled as having acquired disease (6). Brown's syndrome etiologies may include orbital tumors, trauma, surgery, and inflammatory processes involving the trochlea and the superior oblique tendon (7).

Acquired inflammatory Brown's syndrome has been described in a variety of inflammatory arthropathies including: JIA, psoriatic arthritis, post-streptococcal reactive arthritis, and HLA-B27 associated enteropathic arthritis (8-17). Other suspected inflammatory cases may be deemed idiopathic without a clear rheumatic disease associated diagnosis made $(18,19)$. In 2002 Milz et al. performed an immunohistochemical examination of human cadaveric superior oblique tendon including its fibrocartilaginous pully, the trochlea; they identified the presence of aggrecan, link protein, and type 2 collagen in both structures (20). From this finding they speculated that the transient tendonitis flares of Brown's syndrome observed in chronic juvenile arthritis could occur by similar processes understood to occur in rheumatoid arthritis, such as:

1) the cleavage of aggrecan by aggrecanase in articular cartilages;

2) the action of link protein and type 2 collagen as important antigenic targets for autoimmune responses.

Presenting symptoms of acquired inflammatory Brown's syndrome often include diplopia and pain with elevation or adduction. A head tilt or chin-up posture, tenderness to palpation, swelling in the medial periorbital area, and an audible click upon elevation and adduction of the affected eye may be reported (7). These findings were absent in our patient with spondyloarthropathy. The head tilt and chin up posture was present in our patient who later developed oligoarticular JIA. Often they have been described to present in temporal synergy with disease flares of systemic inflammatory disease.

Some feel management of asymptomatic Brown's syndrome should remain conservative (21). Wright's study found that $16 \%$ of acquired Brown's spontaneously resolve within 6-48 months (6). This was the case with our oligoarticular JIA patient; in longitudinal follow up she had stepwise improvement first one side, followed later by the second, with no residual ocular deficiency in motility. We treated our juvenile spondyloarthropathy patient with a tapering oral steroid course and weekly methotrexate, and his Achilles' enthesitis resolved. We presume he had concomitant resolution of his acute ocular tenosynovial inflammation, however a repeat MRI was not performed since his initial study did not 
give enough resolution to assess the underlying process, and this is not uncommon in Brown's syndrome cases $(22,23)$. The persistence of our patient's acquired motility disorder may have been due to his having had long standing active disease, which led to fibrotic scar. An acquired Brown's syndrome patient has been described with an ongoing disorder of ocular movement, despite not having evidence of intrasheath tenosynovitis pathology, but instead perisheath adhesions anterior to the troclea with fibrotic scar around the tendon sheath (6). Since he lacked significant deviation nor diplopia in primary position, steroid injection into the tendon/trochlea complex was not indicated. Biologic therapy inhibiting tumor necrosis factor alpha, would be indicated should he develop objective features of an active, systemically driven tenosynovial inflammatory disease process (24).

We present a case of acquired idiopathic Brown's syndrome in a school-aged HLAB27 positive child with clinical enthesitis. The patient had no lumbar or sacroiliac area pain, rash, gastrointestinal complaints, or signs of intraocular inflammation on slit-lamp exam to help further characterize his HLA-B27-related disorder. His young age at presentation does not allow him to meet strict diagnostic criteria for the JIA subtype enthesitis-related arthritis, and his lack of frank arthritis does not place him within another category of the International League of Associations of Rheumatologists (ILAR) JIA diagnostic criteria (25). Cases of the systemic onset JIA subtype have been described with Brown's syndrome (16). While his preceding history of fever suggested the diagnosis of systemic onset JIA, he did not display rash or the characteristic pro-inflammatory laboratory pattern. An outlying diagnostic consideration was a hereditary periodic fever syndrome such as tumor necrosis factor associated periodic fever syndrome, but we did not feel that clinically there was enough evidence to warrant genetic testing.

Our case of oligoarticular JIA presented with presumed congenital Brown's Syndrome at a young age, years prior to her development of clinical arthritis or to her ophthalmologist's diagnostic appreciation on screening slit lamp examination of anterior iritis. Certainly it is possible that the observed frequency of having both oligoarticular JIA and acquired Brown's syndrome may be no different than having individual unassociated diagnosis. However, since published rates and prevalence data for cases of childhood onset Brown's Syndrome not attributable to orbital tumors, trauma, or surgery are not available, we are unable to accurately determine whether the joint occurrence is more or less than might be expected on the basis of chance. More commonly, acquired Brown's patients present with other rheumatic disease signs or else have a prior understood diagnosis of a chronic arthritis condition. Therefore, in young patients diagnosed with acquired Brown's it is reasonable to perform a careful clinical joint examination for oligoarticular arthritis, especially in female patients in whom oligoarticular JIA is more common.

In summary, we report two children with Brown's syndrome, the first with HLA-B27-positive enthesitis, the second with oligoarticular JIA. These cases exemplify the following points:

1) that HLAB27 spondyloarthroapthy should be considered a diagnostic possibility in a patient presenting with Brown's syndrome and joint pain;

2) that early onset Brown's presenting in young childhood may be acquired and may be the initial presenting feature in a patient with oligoarticular JIA.

In cases of juvenile spondyloarthropathy and JIA, screening for ocular motility disorders, in addition to uveitis, is recommended.

Conflicts of interest: All authors have no commercial or conflicts of interest to disclose.

\section{REFERENCES}

1. Wilson ME, Eustis HS Jr, Parks MM. Brown's syndrome. Surv Ophthalmol. 1989; 34: 153-72. 
2. Scofield RH, Sestak AL. Juvenile spondyloarthropathies. Curr Rheumatol Rep. 2012; 14 : 395-401.

3. Gowdie PJ, Tse SM. Juvenile idiopathic arthritis. Pediatr Clin North Am. 2012; 59: 30127.

4. Brown HW. Congenital structural muscle anomalies. In: Symposium on Strabismus, Trans New Orleans Acad Ophthalmol. St. Louis: CV Mosby. 1950; 205-23.

5. Brown HW. True and simulated superior oblique tendon sheath syndromes. Documenta Ophthalmologica. 1973; 34: 123-36.

6. Wright KW, Silverstein D, Marrone AC, Smith RE. Acquired inflammatory superior oblique tendon sheath syndrome: a clinicopathologic study. Arch Ophthalmol. 1982; 100: 1752-4.

7. Wright KW. Brown's syndrome: diagnosis and management. Trans Am Ophthalmol Soc. 1999; 97: 1023-109.

8. Roifnam CM, Lavi S, Moore AT, Morin DJ, Stein LD, Gelfard EW. Tenosynovitis of superior oblique muscle (Brown's syndrome) associated with juvenile rheumatoid arthritis. J Pediatr. 1985; 106: 617-9.

9. Thorne JE, Volpe NJ, Liu GT. Magnetic resonance imaging of acquired Brown syndrome in a patient with psoriasis. Am J Ophthalmol. 1999; 127: 233-5.

10. Faust AO, Gillenwater JM, Saulsbury FT. Acquired Brown's syndrome in a child with poststreptococcal reactive arthritis. J Rheumatol. 2001; 28: 2748-9.

11. Bradshaw DJ, Bray VJ, Enzenauer RW, Enzenauer RJ, Truwit CL, Damiano TR. Acquired Brown syndrome associated with enteropathic arthropathy: a case report. J Pediatr Ophthalmol Strabismus. 1994; 31: 118-9.

12. Kemp AS, Searle C, Horne S. Transient brown's syndrome in juvenile chronic arthritis. Ann Rheum Dis. 1984; 43: 764-5.

13. Sifuentes Giraldo WA, Morell Hita JL, Bachiller Corral J, Brito Brito E. Acquired Brown's syndrome in a patient with psoriatic arthritis. Reumatol Clin. 2013; 9: 198.

14. Fernandez-Melon J, Munoz-Fernandez S, Hidalgo V, Bonilla-Hernan V, Schlincker A, Fonseca A, et al. Uveitis as the initial clinical manifestation in patients with spondyloarthropathies. J Rheum. 2004; 3: 524-7.
15. Whitefield L, Isenberg DA, Brazier DJ, Forbes J. Acquired Brown's syndrome in systemic lupus erythematosus. Br J Rheumatol. 1995; 34: 1092-4.

16. Moore AT, Morin JD. Bilateral acquired inflammatory Brown's syndrome. J Pediatr Ophthalmol Strabismus. 1985; 22: 26-30.

17. Gezer A, Tuncer S, Canturk S, Kasapoglu E, Cimen AO, Inanc M. Bilateral acquired Brown syndrome in systemic scleroderma. J AAPOS. 2005; 9: 195-7.

18. Can I, Yarangümeli A, Kural G. Brown's syndrome with cyclic characteristic: case report and review of physiopathologic mechanism. J Pediatr Ophthalmol Strabismus. 1995; 32: 243-7.

19. Pronk AE, Demaerel PM, Casteels IK. A 5-year-old boy with acute intermittent acquired Brown's syndrome. Bull Soc Belge Ophtalmol. 2010; (316): 21-6.

20. Milz S, Regner F, Putz R, Benjamin M. Expression of a wide range of extracellular matrix molecules in the tendon and trochlea of the human superior oblique muscle. Invest Ophthalmol Vis Sci. 2002; 43: 1330-4.

21. Lee J. Management of Brown syndrome. Semin Ophthalmol. 2008; 23: 291-3.

22. Bhola R, Rosenbaum AL, Ortube MC, Demer JL. High-resolution magnetic resonance imaging demonstrates varied anatomic abnormalities in Brown syndrome. J AAPOS. 2005; 9: 438-48.

23. Kraft SP, Nabi NU, Wilson ME, Roarty JD, Budning AS, De Faber JT, et al. Bilateral idiopathic Brown's syndrome with delayed onset in the second eye. J AAPOS. 2000; 4: 158-63.

24. Beukelman T, Patkar NM, Saag KG, TollesonRinehart S, Cron RQ, DeWitt EM, et al. 2011 American College of Rheumatology recommendations for the treatment of juvenile idiopathic arthritis: initiation and safety monitoring of therapeutic agents for the treatment of arthritis and systemic features. Arthritis Care Res. 2011; 63: 465-82.

25. Petty RE, Southwood TR, Manners P, Baum J, Glass DN, Goldenberg J, et al. International League of Associations for Rheumatology classification of juvenile idiopathic arthritis: second revision, Edmonton, 2001. J Rheumatol 2004; 31: 390-2. 